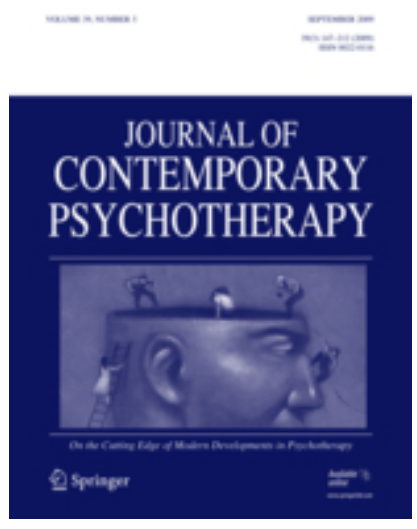

\title{
Virtual reality based treatments in eating disorders and obesity: A review
}

\section{Marta Ferrer-García, José Gutiérrez-Maldonado, Giuseppe Riva (2013)}

DOI: 10.1007/s10879-013-9240-1

The final publication is available at: http://www.springer.com/public+health/journal/10879

\section{ABSTRACT}

Since the late 1990s the use of virtual reality (VR) has expanded in the field of mental disorders. During this time, several VR-based applications have been developed for the treatment of eating disorders (ED) and obesity. Both these pathologies involve disturbances related to eating behavior, weight, and body image. Although effective and well-established protocols are now available for the treatment of ED and obesity, relapses are frequent and a proportion of patients do not improve after intervention. In this context, VR has been shown to be a useful technology for enhancing traditional cognitive-oriented therapies. The main objective of this review was therefore to examine the evidence regarding the efficacy of VR-based treatments as a component of therapeutic interventions for ED and obesity. To this end, three databases (PsycInfo, Medline, and PsycArticles) were searched for the period 1986 to 2012. Only research articles and case studies were selected. Although several methodological deficiencies were detected in the reviewed studies, there is fair evidence for the effectiveness of VR-based treatments in ED and obesity. VR-based interventions usually combine exposure to VR environments with cognitive therapies. The VR component seems to be especially suitable for reducing body image disturbances, such as body image dissatisfaction, and for increasing self-esteem and self-efficacy. The rationale for the use of VR in these areas is also presented and discussed.

Keywords: Virtual reality, treatment, eating disorders, binge eating disorders, obesity, review. 


\section{INTRODUCTION}

These are paradoxical times. While thinness is considered the ideal of beauty in most societies, sedentary lifestyles and overeating have driven obesity to epidemic proportions, its prevalence ranging from $4 \%$ to $36 \%$ depending on the country studied (e.g., Berghöfer et al., 2008; Flegal, Carroll, Kit, \& Ogden, 2012; Thorburn, 2005). Consequently, healthcare professionals must now address two apparently different but, in fact, related concerns associated with weight and eating behavior (Neumark-Sztainer, 2003). On the one hand, the prevalence of obesity is increasing dramatically throughout the world (WHO, 2013), leading to severe health consequences (such as type 2 diabetes, hypertension, and cardiovascular diseases) and high healthcare costs (Kopelman, 2007; Mokdad et al., 2001; Poirier et al., 2006). On the other hand, eating disorders (ED), although much less common than obesity (lifetime prevalence rates for anorexia nervosa, bulimia nervosa, and binge eating disorder are $0.9 \%, 1.5 \%$, and $3.5 \%$ among women, and $0.3 \%$, 0.5\%, and 2.0\% among men; Hudson, Hiripi, Pope, \& Kessler, 2007), are also linked to other psychopathologies, medical disorders, and impaired functioning at school, work, in relationships, or in other social roles.

Although ED and obesity are considered to be very different disorders, they actually share many features. Obviously, they are both weight-related problems. ED patients typically move from anorexia nervosa (AN) to bulimia nervosa $(\mathrm{BN})$ or binge eating disorder (BED), while patients with $\mathrm{BN}$ and, especially, BED are frequently overweight and even obese. Body dissatisfaction and unhealthy dieting practices are risk factors for the development of both ED and obesity (Burrows \& Cooper, 2002; Davison, Markey, \& Birch, 2000; Haines \& Neumark-Sztainer, 2006; Vander Wal \& Thelen, 2000). Moreover, binge eating behavior is common in patients with ED and in people with obesity (Yanovski, 2002). The aforementioned eating-related disorders are also comorbid with disorders of mood and anxiety (Goodman \& Whitaker, 2002; Lumeng, Gannon, Cabral, Frank, \& Zuckerman, 2003).

There are multiple factors associated with the onset and maintenance of obesity and ED and which complicate their treatment and make an individualized patient-oriented approach necessary. Cognitive behavioral therapy (CBT) has been established as the method of choice for the treatment of BN and BED, it having been shown to lead to significant improvements in attitudes and behaviors related to ED, as well as reducing the associated symptoms, such as depression (Fairburn, 2012). CBT has also been widely used to treat AN and EDNOS (eating disorder not otherwise specified), although more research on its 
effectiveness is needed (Fairburn et al., 2007; Wilson, Grilo, \& Vitousek, 2007). Despite the usefulness of this approach, some patients do not improve after intervention, and others will relapse. Something similar occurs with obesity treatment: Even when intervention is successful in the short term (at the end of treatment), weight management efforts are often ineffective over the long term (at follow-up) (Jeffery et al., 2000; Mann et al., 2007). Consequently, over the last couple of decades, a growing body of research has begun to examine the extent to which the use of advanced technologies such as virtual reality (VR) may enhance traditional intervention methods.

Virtual reality has proved useful for the assessment and treatment of several mental disorders, mainly anxiety disorders (Powers \& Emmelkamp, 2008), and it is especially suitable as an exposure method. In fact, exposure to virtual environments produces emotional and behavioral responses similar to those that occur in the real world, and studies show that VR exposure is as effective as in vivo exposure (Gorini, Griez, Petrova, \& Riva, 2010; Ferrer-García, Gutiérrez-Maldonado, Caqueo-Urízar, \& Moreno, 2009; Gutiérrez-Maldonado, Ferrer-García, Caqueo-Urízar, \& Moreno-Pérez, 2010; Powers \& Emmelkamp , 2008). As a result, VR technology has been used in the treatment of most anxiety disorders, and its application has been extended to the treatment of a wide range of psychopathologies (Botella et al., 2004; Côté \& Bouchard, 2008; Cukor, Spitalnick, Difede, Rizzo, \& Rothbaum, 2009; Gorini \& Riva, 2008; Krijn, Emmelkamp, Olafson, \& Biemond, 2004; Maher \& Gold, 2009; Parsons \& Rizzo, 2008; Riva, 2005; Riva et al., 2009).

Virtual reality enables the creation of virtual scenarios that are very similar to those of the real world. This allows strict control of situational parameters while maintaining high ecological validity. VR also provides a safe and flexible environment in which users may face feared situations at their own pace, reducing their resistance to accept exposure to the feared situation. Other advantages are that it allows self-training, it enables therapists to conduct treatments in which it is impossible or very difficult to apply in vivo exposure (e.g., flying phobia), and it offers a greater degree of confidentiality, since treatment takes place in the practitioner's office. Finally, compared with imagery exposure, VR is much more immersive, as it stimulates several sensory modalities (auditory, visual, and vestibular). This makes it useful for participants who find it difficult to imagine scenes, and it facilitates their emotional involvement. 
The pioneering studies on the application of VR for the treatment of ED were conducted by Riva and colleagues (Riva, Melis, \& Bolzoni, 1997) in the context of the European VREPAR PROJECT (Virtual Reality Environments for the Psychoneurophysiological Assessment and Rehabilitation Project). This research group (Riva \& Melis, 1997a; 1997b; Riva et al., 1997) developed the VEBIM (Virtual Environment for Body Image Modification), composed of five virtual reality environments for the treatment of body image disturbance. Zone 1 (Training room and weighing balance) and zone 2 (Kitchen and office) are used for training users in navigation and interaction skills in the virtual environments, as well as for focusing attention on weight, eating, and food choice. In both the kitchen and the office, the user can eat and drink by touching an item with the mouse, after which the corresponding caloric intake is recorded. Based on the caloric intake, the balance shows participants the changes they have experienced in weight after eating. In zone 3, users are exposed to pictures of models which are used as stimuli to support a cognitive approach: Feelings and beliefs elicited by the images are identified and discussed. In zone 4 (Mirror room), users are confronted with photographs of their real bodies, previously digitalized. Viewing their own bodies also elicits feelings and distorted beliefs that are then discussed during the session. Users are instructed to imagine their bodies as being different to how they are on several dimensions (size, race, age, etc.), as well as before and after eating or before and after social/academic/job successes and failures. Finally, zone 5 consists of a room with four doors of different dimensions. Users have to choose the door that corresponds exactly to their width, the aim being to improve awareness of body image distortion. Socratic questioning is used during exposure in order to help patients to draw appropriate conclusions from the experience (Riva et al., 1998). For a more detailed explanation of virtual reality environments, see Riva (1998).

The original VEBIM has undergone several updates over the last ten years (VEBIM 2; VREDIM). The current version is based on the NeuroVR (Riva et al., 2009; Riva et al., 2011; Riva et al., 2007) open source software (http://www.neurovr.org) and is used in 10 biweekly immersive virtual reality sessions within the context of cognitive behavioral treatment for obesity and eating disorders. NeuroVR includes 14 virtual environments that are used by the therapist during a 60 -min session with the patient. These environments present critical situations (home, supermarket, pub, restaurant, swimming pool, beach, and gymnasium) related to maintenance/relapse mechanisms, as well as two body image comparison areas. Through the VR experience, patients practice both eating/emotional/relational management and general decision-making and problem-solving skills. By directly practicing these skills within the VR 
environment, patients are helped to develop specific strategies for avoiding and/or coping with critical situations related to the specific weight problem. The first session is used to assess any stimuli that might elicit abnormal eating behavior. Specifically, attention is focused on the patient's concerns about food, eating, shape, and weight. The next nine sessions are used to assess and modify expectations and emotions related to food and weight (functional analysis), the strategies used to cope with difficult interpersonal and potential maintenance situations, and the individual's body experience. The intrinsic characteristics of the VR experience (a distorted egocentric viewpoint) make it easier for the cognitive system to access unconscious information related to body schema (perceptual model of the body: integration of postural, tactile, visual, kinesthetic, and vestibular information). In fact, during immersive VR exposure, discrepancies between the signals arriving from the user's proprioceptive system and the visual signals from the virtual environment alter body perceptions. Although this phenomenon may have undesirable consequences such as discomfort or simulator sickness (a form of motion sickness that produce symptoms such as lightheadedness or blurred vision), it also leads to a greater awareness of the associated sensorimotor and perceptual processes. As Gallagher (1995) found, changes produced in several aspects of body schema may lead to changes in the individual's body image.

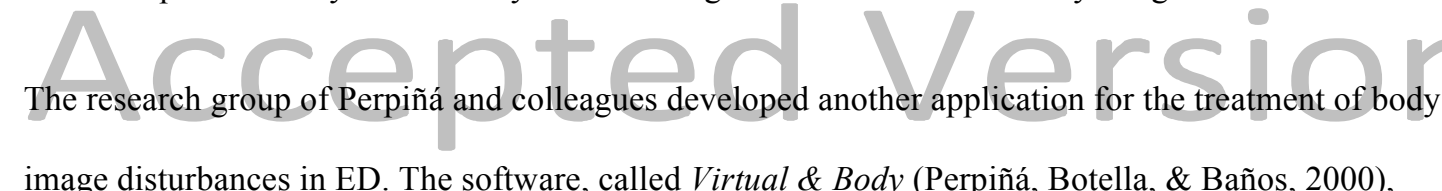
includes six virtual reality environments. Scenario 1 (Learning room) is a training environment in which the user becomes familiar with the system. In scenario 2 (Virtual scales and kitchen), the discrepancy indexes of body weight (real, subjective, desired, and healthy) are recorded and discussed with the user before and after "virtually" eating different kinds of food. In scenario 3 (Exhibit room), users are exposed to photographs of underweight, normal weight, and overweight people. The main objective of this environment is to train users to manage the concept of body mass index (BMI) and aspects related to social comparison. In scenario 4 (Two-mirror room), perceptual body image distortion is analyzed and treated. Users model their perceived body image using a 3D figure and contrast this modeled figure with both their real and ideal body image. The appropriate corrections are made and discussed with the patient. In scenario 5 (Your body in space), body image distortion is again assessed and discussed. In this environment, users have to decide which space they would need to free up in order to enable their virtual body to pass through a door. Finally, scenario 6 (Mirrored room) allows participants to modify several 3D human figures in order to represent, for example, their perceived body, their ideal body, and their healthy 
body. Note that the two-mirror and mirrored rooms are also used as a tool for the assessment of body image disturbances. The team behind this software (Perpiñá, Botella, \& Baños, 2003) argue that the main advantages of VR are: First, it allows the user to model and reflect his/her body image, that is, a mental representation; second, it enables patients to face and deal with their body image disturbances; third, VR is an objective judge, and hence the information provided to users regarding their body image is accepted with less resistance than is information provided by health staff; and fourth, this technology allows users to face their fears in a protected environment before being exposed to the real situation. All this increases their motivation to engage in treatment.

Recently, we reviewed the published literature on the use of VR for the study, assessment, and treatment of body image disturbance in ED, in both non-clinical and clinical populations (Ferrer-García \& Gutiérrez-Maldonado, 2012). Our purpose now is to extend this review to obesity, which shares several features with ED, and to focus on treatment in clinical populations. Thus, the main objective of this review is to examine the evidence for the efficacy of VR-based treatments as a component of therapeutic interventions for $\mathrm{ED}, \mathrm{BED}$, and obesity.

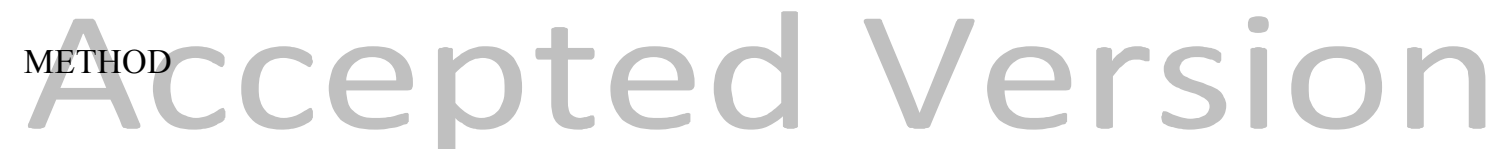

A search of three databases (PsycInfo, Medline, and PsycArticles) was conducted using the following terms and Boolean operators: [(obesity or eating disorders or anorexia or bulimia or binge eating or EDNOS) and (Virtual reality) in abstracts]. In this initial search we identified and screened 87 abstracts. Each abstract was systematically reviewed in order to be sure that the article fitted with the objectives of the study. Our inclusion criteria (Table 1) were broad and covered the period from 1986, the year in which the term virtual reality was coined by Lanier (Zimmerman, Lanier, Blanchard, Bryson, \& Harvill, 1986), to 2012.

\section{INSERT TABLE 1 HERE}

Only research articles and case studies were selected. Reviews, book chapters, dissertations/theses, and conference presentations were excluded. 
We identified 17 studies in 15 articles addressing the efficacy of VR-based treatment for ED, BED, and obesity (Table 2). Four were case studies (all addressing ED), five were non-controlled studies (three addressing ED and two obesity), and 8 were controlled studies (four addressing ED and four obesity). Body image disturbance was the main target of the VR component in these interventions, and body dissatisfaction was the primary outcome variable assessed. Other outcomes often included were depression, self-esteem, assertiveness, and other symptoms related to ED.

\section{INSERT TABLE 2 HERE}

Two general facts emerged from this review. Firstly, only two main research groups have used VR technology in the treatment of ED, obesity, and other related pathologies: One is the group of Riva in Milan (Italy) and the other the group of Perpiñá in Castellón and Valencia (Spain). Both groups use VR to improve cognitive-behavioral therapy, and they have also developed VR-based software for the assessment and treatment of body image disturbances. The second fact is that randomized controlled studies involving clinical populations are scarce, although results are encouraging.

\section{Efficacy of VR-based treatments as a component of therapeutic interventions for ED} (anorexia nervosa, bulimia nervosa, and binge eating disorder)

Riva and colleagues (Riva, Bacchetta, Barufi, Rinaldi, \& Molinari, 1998, 1999) were the first to publish evidence about the usefulness of a VR-based cognitive-behavioral treatment for improving body satisfaction. A 22-year-old female with AN binge-eating/purging subtype was treated for eight weeks using experiential cognitive therapy, an intervention approach that combined cognitive-behavioral treatment with the use of VR technology (VEBIM 2). At the end of treatment the patient showed greater body awareness, decreased body dissatisfaction, and fewer avoidance behaviors and grooming habits associated with a negative body image. The patient also presented greater motivation to change. Perpiñá and colleagues (Perpiñá, Baños, Botella, \& Marco, 2001) also reported the case of an ED patient whose symptoms improved after a VR-based treatment. The patient, a 22-year-old woman with AN, showed an important reduction in body dissatisfaction, in negative thoughts and attitudes related to body image, in the drive for thinness, and a the number of situations associated with body discomfort. These achievements had even increased at one-year follow-up. Salorio del Moral and colleagues (Salorio del Moral et al., 2004) combined 15 group CBT sessions with 10 individual VR exposure sessions (Perpiñá et al., 2000) in order to treat a 15-year-old female with restrictive AN. After treatment the patient showed 
greater body satisfaction, increased interoceptive awareness, and a decreased tendency toward perfectionism and asceticism. Furthermore, at one-year follow-up there were no disturbances in either body image or weight: The patient showed even greater body satisfaction and interpersonal trust, and also presented decreased perfectionism, a weaker tendency toward asceticism, less social insecurity, and no drive for thinness.

Recently, another case study was published in which VR was used not for treating body image disturbances but to treat food avoidance and associated negative emotions and thoughts (Cardi et al., 2012). A 21-year-old patient with restrictive AN was given a VR module in addition to the Maudsley Model of Treatment for Adults with Anorexia Nervosa (MANTRA). As the patient reported intense fears and anxiety related to food, VR was used as an exposure technique for treating this phobic behavior. The VR software displayed a virtual kitchen with food of different caloric contents that the user could "cook" and "eat". During the first session the patient was asked to build a hierarchy of feared food (from the least to the most frightening). The following sessions consisted of gradual exposure to the food items. By the end of the VR module the patient had reduced the number of safety behaviors (such as cutting food into small pieces or eating quickly), as well as her anxiety, fear, and guilt feelings related to food exposure. She also increased the number of food choices in real life and improved the relationship with her family and her social life. Eating symptoms, depression, and anxiety were all reduced, while self-esteem and confidence to change were increased. Furthermore, when asked for feedback regarding the VR experience, she reported a maximum score of enjoyment and helpfulness.

Our review identified three non-controlled studies, all of which found that VR-based therapy can induce positive changes in body image and its associated behaviors in overweight patients with ED. Twenty-five women with BED (Riva, Bacchetta, Baruffi, Cirillo, \& Molinari, 2000; Riva, Bacchetta, Baruffi, Rinaldi, Vincelly, \& Molinari, 2000) and 14 women with EDNOS (Riva, Bacchetta, Baruffi, Cirillo, \& Molinari, 2000), all of whom were seeking treatment at the Weight Reduction Unit of the Istituto Auxologico Italiano (Verbania, Italy), were administered the VEBIM 2, a VR-based treatment for body image disturbance. Participants simultaneously attended an in-patient weight reduction program. In both samples, participants improved their overall body satisfaction and reduced associated problematic eating and social behaviors. Despite the positive outcomes obtained in these studies, methodological limitations such as the lack of a control or comparison group mean that the findings should be considered as preliminary. 
The first controlled study using VR technology for the treatment of ED was conducted at the end of the 1990s by Perpiñá and colleagues (Perpiñá et al., 1999). Eighteen ED outpatients were randomly assigned to one of two treatments: the VR condition (cognitive-behavioral treatment plus VR exposure) or the standard treatment for body image disturbances (cognitive-behavioral treatment plus relaxation). All of the 13 patients who completed treatment improved significantly. However, those who were treated with the VR component showed a significantly greater improvement in general psychopathology, ED-related symptoms, and specific body image variables (highest level of satisfaction with their body in social situations, fewer thoughts and negative attitudes towards the body, less afraid of their weight, and less fearful of achieving a healthy weight). Furthermore, dropout rate was lower in the VR group. These results show that greater improvement was achieved through the addition of the VR component to the standard body image treatment. Consequently, the researchers offered patients in the standard treatment group the possibility of being treated with the VR component as well. The sample in this second study comprised 12 patients ( 7 with AN and 5 with BN). The results showed that the improvement achieved after completing the treatment was not only maintained after 12 months but actually increased (Perpiñá, Marco, Botella, \& Baños, 2004).

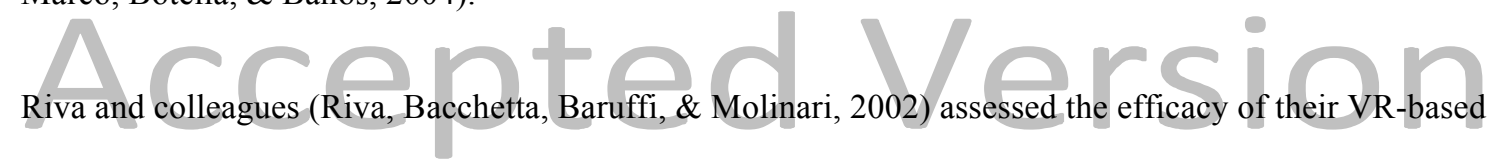
multidimensional approach to body image disturbances (VREDIM) in 20 BED patients. The sample was randomly divided into two groups: the experimental group (VREDIM plus low-calorie diet and physical exercise) and the control group (cognitive-behavioral nutritional group plus low-calorie diet and physical exercise). After treatment, all patients had stopped their binging behavior. However, participants in the experimental group reduced significantly their levels of body dissatisfaction and anxiety, and significantly increased self-efficacy and motivation to change. Moreover, they reported a significantly more realistic ideal body at the end of the treatment. By contrast, participants in the control group only showed a significant reduction in anxiety after treatment.

In a similar study, Riva's group (Riva, Bacchetta, Cesa, Conti, \& Molinari, 2003) randomly divided a sample of 36 women with binge eating disorder into four groups according to the type of treatment received: experiential cognitive therapy including VR (ExCT group), cognitive-behavioral therapy alone (CBT group), nutritional psychoeducational group, and a waiting list (no treatment). At six-month followup $77 \%$ of participants in the ExCT group had stopped bingeing, compared with $56 \%$ and $22 \%$ in the 
CBT and nutritional psychoeducational groups, respectively. Moreover, ExCT was more effective than the other treatments in improving body image satisfaction, self-esteem, and resistance to social pressure.

\section{Efficacy of VR-based treatments as a component of therapeutic interventions for obesity}

Virtual reality has also been used as a component in the treatment of body image disturbance in obesity. In 2000, Riva and his team (Riva, Bacchetta, Baruffi, Cirillo, \& Molinari, 2000; Riva, Bacchetta, Baruffi, Rinaldi, Vincelli, \& Molinari, 2000) tested the efficacy of VR in modifying body image disturbances (body image distortion and body image dissatisfaction) in patients with this pathology. Eighteen women with obesity (BMI>35) who contacted the Weight Reduction Unit of the Istituto Auxologico Italiano were assessed before and after being treated by means of a brief VR therapy consisting of five fortnightly sessions. Two major results emerged from this study. First, there was no simulator sickness, even though the entire sample was composed of women, who are especially susceptible to such alterations (Griffin, 1990). This finding supports the possibility of using VR for therapeutic purposes without fear of unpleasant side effects. Secondly, the measures used to assess body image disturbances showed a significant post-treatment improvement: Patients showed a reduction in body image dissatisfaction and a more realistic perception of their body. Furthermore, the authors note that this improvement led, in turn, to a reduction in disturbed behaviors, such as the use of disguising clothes, and to an improvement in social activities. They conclude that despite being a preliminary study this research paves the way for the use of VR in developing effective short-term interventions to treat obesity and other related pathologies.

On account of the promising results of previous studies, Riva and colleagues conducted several controlled studies over the following decade. In 2001, they (Riva, Bacchetta, Baruffi, \& Molinari, 2001) divided 28 women with obesity $(\mathrm{BMI} \geq 35)$ into two equal groups in order to compare the results obtained from the application of two different treatments: one based on VR (VREDIM) and another on the psychonutritional intervention of traditional cognitive-behavioral treatment (PNI). Both groups also took part in a parallel diet and exercise program. All patients reduced their weight after treatment, and although this reduction was greater in the VREDIM group the difference was not significant. However, participants in the experimental group significantly reduced their level of body dissatisfaction, anxiety, and overeating, and also presented significantly increased self-efficacy. By contrast, women in the control group only showed a significant reduction in anxiety and an increase in exercising. 
In 2006, the Milan team (Riva et al., 2006) provided new evidence for the efficacy of ExCT in the treatment of body image disturbance in obesity. On this occasion the sample comprised 211 women with obesity (aged 18 to 50 and with BMI over 40) but without any other serious psychiatric disorders, a further common feature being that they had all experienced at least one failure when following a previous obesity treatment. The women were randomly assigned to one of three groups: ExCT, traditional CBT, or nutritional psychoeducation. As in previous studies, the ExCT group not only obtained better outcomes than did the other two groups in terms of reduced body dissatisfaction and increased self-efficacy, but they also had fewer relapses during the six-month follow-up. The advantage of ExCT in these patients is that during the VR exposure they experience critical situations related to relapse in real life. In virtual environments the user can face and learn to cope in such situations, and this, in addition to allowing the body image disturbance to be treated, increases the user's sense of self-efficacy. Although the follow-up period was short, this study used a broad randomized sample, which gives strength and credibility to the results obtained.

More recently, Riva's group has explored other uses of VR technology as a component in obesity treatment. For instance, Manzoni and colleagues (Manzoni et al., 2008) assessed the efficacy of a 3-week, VR-enhanced relaxation training protocol in reducing emotional eating, anxiety, and depression in a sample of 60 females with obesity. All participants in the trial underwent a 5-week hospital-based program for weight reduction and rehabilitation, with relaxation protocols being administered during the inpatient program. The sample was randomly divided into three conditions: the VR condition, the imagery condition, and the control group, who did not receive a relaxation program. In the VR-enhanced protocol, narratives for relaxation were presented together with the Green Valley, a highly relaxing virtual environment. In the imagery protocol, narratives suggested imagining a similar environment. After treatment, patients in both experimental conditions (VR and imagery conditions) showed reduced levels of depression and anxiety, as well as greater perceived self-efficacy for eating control. These changes did not occur in the control group. However, patients in all three groups reduced weight significantly and reported no further emotional eating episodes. At three-month follow-up, control participants showed no significant change, whereas the experimental groups showed significant improvements on all assessed variables. The VR condition produced a greater reduction in emotional eating than did the imagery condition (Manzoni et al., 2009).

\section{DISCUSSION AND CONCLUSIONS}


Eating disorders and obesity constitute severe health problems in Western society. Although they have frequently been considered different pathologies, they are both disturbances related to eating behavior, weight, and body image. Despite there being well-established interventions that have proved effective for the treatment of these pathologies, relapses are frequent and a proportion of patients do not improve after intervention. In this context, VR is a suitable technology for enhancing traditional cognitive-oriented therapies.

Virtual reality has proven to be a useful technology in the study, assessment, and treatment of mental disorders. Indeed, several studies, especially in the field of anxiety disorders, provide evidence of its effectiveness. Research on the application of this technology in the treatment of ED and obesity began more than 20 years ago. Since then, several VR applications have been developed for use in conjunction with traditional treatments, and the effectiveness of this software has been tested in case studies, as well as in non-controlled and controlled studies.

Several conclusions can be drawn from this review of the literature in the field. Firstly, most of the research on the use of VR in the treatment of ED and obesity has been conducted by two groups: that of Riva in Italy and the group of Perpiñá, Botella, and Baños in Spain. Secondly, VR-based interventions usually combine exposure to VR environments with components based on cognitive therapy. Although the first VR-based treatments were developed specifically to address body image disturbance, the literature shows that they can also influence other aspects such as perceived self-efficacy. More recent studies have extended the use of the VR component to the treatment of other symptoms related to disturbed eating behavior, such as anxiety or distress in emotional eating (Manzoni, et al., 2009). In these cases, VR is used to enhance relaxation protocols. A third conclusion that emerges is that cases of simulator sickness associated with exposure to VR environments are rare; such cases are usually characterized by ocular problems, such as eye-strain, blurred vision, and fatigue, as well as disorientation and balance disturbances, and nausea (Riva, Bacchetta, Baruffi, \& Molinari, 2001). Fourthly, all the reviewed studies report positive results regarding the effectiveness of adding VR to the standard intervention. The VR component seems to be especially suitable for the treatment of body image disturbance and related behaviors (such as avoidance and grooming behaviors), of self-esteem and selfefficacy, and of disturbed emotions such as depression. However, a critical question that needs to be considered if this technology is to become more widely used in the treatment of eating disorders and 
obesity concerns the rationale: Why is virtual reality effective in dealing with these aspects of eatingrelated disturbances?

The first point to make is that VR is an experiential tool whose features enable it to support empowerment for change in patients with ED and obesity. Applied to the field of health, the idea of empowerment involves increasing one's authority and control over the resources and decisions that affect one's health. ED patients and people with obesity typically feel ambivalent about the idea of change for better health (since any change involves pros and cons), and this ambivalence can leave them stuck for long periods of time. Moreover, some patients do not have the skills, assertiveness, or self-esteem required to make a real choice and to be able to change (Riva et al, 2009). The value of virtual reality here is that it is a special, sheltered setting in which patients can start to explore and act without feeling threatened (Botella, Perpiñá, Baños, \& García-Palacios, 1998). Nothing the patient fears can "really" happen to them in VR, and with such an assurance they can freely experiment and experience feelings and thoughts. As discussed before, the exposure to virtual environments produces emotional and behavioral responses similar to those that occur in the real world, and studies show that VR exposure is as effective as in vivo exposure (Gorini, Griez, Petrova, \& Riva, 2010; Ferrer-García, Gutiérrez-Maldonado, Caqueo-Urízar, \& Moreno, 2009; Gutiérrez-Maldonado, Ferrer-García, Caqueo-Urízar, \& Moreno-Pérez, 2010; Powers \& Emmelkamp , 2008). Moreover, VR is not only a good tool to re-create situations but also a safe place where patients learn to challenge and cope with distorted mental representations of their fears. As underlined by Glantz, "cognitively oriented therapists challenge assumptions in a variety of ways [...]. Nevertheless, people often have a hard time giving up their assumptions, no matter how self-defeating. One reason it is so difficult to get people to update their assumptions is that change often requires a prior step - recognizing the distinction between an assumption and a perception. [...] We anticipate using VR to help people in distress make the distinction between assumptions and perceptions." (Glantz, Durlach, Barnett, \& Aviles, 1997, p. 96).

A second point is that VR can be considered an "embodied technology", due to its effects on body perceptions (Spagnolli \& Gamberini, 2005). A number of studies have demonstrated that it is possible to use VR to induce controlled changes in the experience of the body (Lenggenhager, Tadi, Metzinger, \& Blanke, 2007; Riva et al., 2000; Slater, Spanlang, Sanchez-Vives, \& Blanke, 2010), and this is particularly relevant given the role of body image disturbances in ED and obesity. Recently, Riva and colleagues proposed the allocentric lock hypothesis (Gaudio \& Riva, 2013; Riva, 2007, 2011, 2012; Riva 
et al., 2012; Riva \& Gaudio, 2012), suggesting that ED and obesity may be the outcome of a primary disturbance in the way the body is experienced and remembered: Individuals with these disorders may be locked into an allocentric (observer view) image schema of their body that is no longer updated by contrasting egocentric representations driven by perception. Although this hypothesis may appear more suitable as a description of eating disorders than of obesity, research does support a lack of a direct correlation between weight loss and body image (Sorbara \& Geliebter, 2002; Dalle Grave et al., 2007). For example, a common problem after bariatric surgery is that patients remain unhappy about their weight, despite a successful treatment. As a general guideline, bariatric surgery is considered successful when $50 \%$ of excess weight is lost and this weight loss is sustained for up to five years. However, most patients with obesity have different expectations. In a study by Kaly and colleagues (2008), 284 bariatric patients stated that they would be "happy" only after a $77 \% \pm 9 \%$ excess body weight loss, with a loss of $67 \% \pm 10 \%$ excess body weight being considered "acceptable". A $49 \% \pm 14 \%$ excess body weight loss, the gold standard for clinicians, was regarded as "disappointing". Importantly, expectations of this kind are reported both before (Kaly et al., 2008) and after surgery (Riva et al., 2012). In order to explore this issue, Guardia and colleagues (2013) evaluated body image in a bariatric patient after successful surgery (weight loss from $125 \mathrm{~kg}$ (BMI 45.9) to $65 \mathrm{~kg}$ ) and found a distortion in both implicit and explicit representations of the body. For example, in the aperture task (an implicit task based on the question: “could you walk through this aperture without turning sideways?”), the patient significantly overestimated her "passability" — as if her body was larger than it really was. To put it simply, these patients cannot win: Whatever they may do to modify their real body, including successful bariatric surgery leading to significant weight loss, they will always be trapped in a virtual body (allocentric image schema) that they hate. This situation usually has one of two effects: Either subjects turn to more radical dietary restraint, or they decide to stop any form of food control and engage in "disinhibited" eating behaviors. The shift from a locked allocentric representation to an eating or weight disorder may be explained by social influence, since the media and culture promote dieting as the best way to improve one's body image satisfaction. However, the impossibility of improving body image, even after a demanding diet, locks the patient into an unsatisfying body. A recent clinical trial involving 163 women with morbid obesity used VR as a way of unlocking this image schema and found that this approach was significantly better at one-year follow-up in maintaining the results of the treatment, as compared with both CBT and nutritional treatment (Riva et al, 2012). 
Despite a strong rationale for the use of VR in this context, and the good experimental results obtained, there are several methodological deficiencies in the reviewed literature: Only eight randomized controlled studies have been found, sample sizes are sometimes insufficient for drawing conclusions about differential efficacy across assessed treatments, and not all the studies report follow-up data. These deficiencies are more marked in ED studies, whereas the controlled studies involving patients with obesity are acceptable in terms of their methodology. In order to evaluate the quality of the reviewed literature as a whole, we applied the criteria developed by Greer and colleagues (Greer, Mosser, Logant, \& Halaas, 2000, p. 708). These authors assess three domains: quality of research, number of studies and adequacy of sample size, and consistency of findings. The assessment of these domains yields a classification based on four categories: good evidence (grade I), fair evidence (grade II), limited evidence (grade III), and no evidence available or conclusions supported only by opinion (grade IV). Applying this classification, it can be concluded that, despite the aforementioned limitations, there is fair evidence for the effectiveness of VR-based treatments in relation to body image disturbance in ED and obesity.

Taken together, these results should pave the way for a more widespread use of VR technology in the treatment of ED and obesity, specifically as part of multi-component interventions based on behavioral and cognitive therapies. Among the proposed applications of VR in this field we have already mentioned the use of virtual environments as a relaxation method to complement the treatment of both ED and obesity (Manzoni et al., 2009). Then there is the case of exergames (video games that also involve exercise), which have recently been used for health education and obesity prevention (Barros, Neves, Correira, \& Soares, 2012; Staiano \& Calvert, 2011). Finally, several authors have highlighted the similarities between the craving experience in addictive disorders and the hunger shown by patients with binge eating and obesity (for example, Riva et al., 2006). In this context, VR could be used as a cueexposure treatment to increase self-control related to eating behavior (Bordnick, Carter, \& Traylor, 2011). In sum, not only is there sufficient evidence to support the effectiveness of VR-based treatments for ED and obesity but, furthermore, there is a wide range of applications of this technology that need to be explored and developed.

\section{ACKNOWLEDGMENTS}

This study was supported by the Spanish Ministry of Science and Innovation (Project PSI2011-28801: "Virtual Reality Cue Exposure Treatment for Bulimia Nervosa") 


\section{REFERENCES}

Barros, M., Neves, A., Correira, W., \& Soares, M. (2012). Exergames: The role of ergonomics and design in helping to control childhood obesity through physical and functional exercise program. Work: A Journal of Prevention, Assessment and Rehabilitation, 41, 1208-1211.

Berghöfer, A., Pischon, T., Reinhold, T., Aprovian, C.M., Sharma, A.M., \& Willich, S. (2008). Obesity prevalence from a European perspective: a systematic review. BMC Public Health, 8(200). doi:10.1186/1471-2458-8-200.

Bordnick, P.S., Carter, B.L., \& Traylor, A.C. (2011). What virtual reality research in addictions can tell us about the future of obesity assessment and treatment. Journal of Diabetes Science and Technology, 5(2), 265-271.

Botella, C., Perpiñá, C., Baños, R.M., García-Palacios, A. (1998) Virtual reality: a new clinical setting lab. Studies in Health Technology and Informatics, 58, 73-81.

Botella, C., Quero, S., Baños, R., Perpiñá, C., García-Palacios, A., \& Riva, G. (2004) Virtual Reality and Psychotherapy. In G. Riva, C. Botella, P. Legeron, G. Optale (Eds.), Cybertherapy, Internet and Virtual Reality as Assessment and Rehabilitation Tools for Clinical Psychology and Neuroscience (pp. 37-54). Amsterdam. IOS Press.

Burrows, A., \& Cooper, M. (2002). Possible Risk Factors in the Development of Eating Disorders in Overweight Pre-Adolescent Girls. International Journal of Obesity and Related Metabolic Disorders, 26(9), 1268-1273.

Cardi, V., Krug, I., Perpiñá, C., Mataix-Cols, D., Roncero, M., \&Treasure, J. (2012), The Use of a Nonimmersive Virtual Reality Programme in Anorexia Nervosa: A Single Case-Report. European Eating Disorders Review, 20, 240-245.

Côté, S., \& Bouchard, S. (2008). Virtual reality exposure's efficacy in the treatment of specific phobias: A critical review. Journal of Cybertherapy and Rehabilitation, 1(1), 75-91.

Cukor, J., Spitalnick, J., Difede, J. A., Rizzo, A., \& Rothbaum, B. O. (2009). Emerging treatments for PTSD. Clinical Psychology Review, doi: 10.1016/j.cpr.2009.09.001. 
Dalle Grave, R., Cuzzolaro, M., Calugi, S., Tomasi, F., Temperilli, F., \& Marchesini, G. (2007). The effect of obesity management on body image in patients seeking treatment at medical centers. Obesity, 15(9), 2320-2327.

Davison, K.K., Markey, C.N., \& Birch, L.L. (2000). Etiology of Body Dissatisfaction and Weight Concerns Among 5-year-old Girls. Appetite, 35(2), 143-151.

Fairburn, C. G. (2012). El tratamiento de los problemas de atracones. In C. Fairburn (Ed). La Superación de los Atracones de Comida (pp. 155-174). Barcelona: Paidós.

Fairburn, C. G., Cooper, Z., Bohn, K., O’Connor, M.E., Doll, H. A., \& Palmer, R. L. (2007). The severity and status of eating disorder NOS: implications for DSM-V. Behaviour Research and Therapy, $45,1705-1715$.

Ferrer-García, M., Gutiérrez-Maldonado, J.(2012). The use of virtual reality in the study, assessment, and treatment of body image in eating disorders and nonclinical samples: a review of the literature. Body Image, 9(1), 1-11

Ferrer-García, M., Gutiérrez-Maldonado, J., Caqueo-Urízar, A., \& Moreno, E. (2009). The validity of virtual environments for eliciting emotional responses in patients with eating disorders and in controls. Behavior Modification, 3, 830-854.

Flegal, K.M., Carroll, M.D., Kit, B.K., \& Ogden, C.L. (2012). Prevalence of obesity and trends in the distribution of body mass index among US adults, 1999-2010. The Journal of the Medical Association, 307, 491-497.

Gallagher, S. (1995). Body schema and intentionality. In J.L. Bermúdez, A. Marcel, \& N. Eilan (Eds), The Body and the Self. Cambridge (MA): MIT Press.

Gaudio, S., \& Riva, G. (2013). Body Image Disturbances in Anorexia: The link between functional connectivity alterations and reference frames. Biological Psychiatry, 73(9), 25-26.

Glantz, K., Durlach, N.I., Barnett, R.C., \& Aviles, W.A. (1997) Virtual reality (VR) and psychotherapy: Opportunities and challenges. Presence, Teleoperators, and Virtual Environments, 6(1), 87105. 
Goodman, E., \& Whitaker, R. A. (2002). Prospective Study of the Role of Depression in the Development and Persistence of Adolescent Obesity. Pediatrics, 110(3), 497-504.

Gorini A., Griez E., Petrova A., \& Riva G. (2010). Assessment of the emotional responses produced by exposure to real food, virtual food and photographs of food in patients affected by eating disorders. Annals of General Psychiatry, 9(30). doi:10.1186/1744-859X-9-30.

Gorini, A., \& Riva, G. (2008). Virtual reality in anxiety disorders: the past and the future. Expert Reviews Neurotherapeutics, 8(2), 1-19.

Greer, N., Mosser, G., Logan, G., \& Halaas, G.W. (2000). A practical approach to evidence grading. The Joint Commission Journal on Quality Improvement. 26(12), 700-712.

Griffin, M. J. (1990). Handbook of human vibration. London: Academic Press.

Guardia, D., Metral, M., Pigeyre, M., Bauwens, I., Cottencin, O., \& Luyat, M. (2013). Body distortions after massive weight loss: lack of updating of the body schema hypothesis. Eating and Weight Disorders - Studies on Anorexia, Bulimia and Obesity, 1-4. DOI: 10.1007/s40519-013-0032-0

Gutiérrez-Maldonado, J, Ferrer-García, M., Caqueo-Urízar, A., \& Moreno, E. (2010). Body image in eating disorders: The influence of exposure to virtual environments. CyberPsychology \& Behavior. 13(5), 521-531

Haines, J., \& Neumark-Sztainer, D. (2006). Prevention of obesity and eating disorders: a consideration of shared risk factors. Health Education Research, 21(6), 770-782.

Hudson,J. I., Hiripi, E., Pope, H.G., \& Kessler, R.C. (2007). The prevalence and correlates of eating disorders in the national comorbidity survey replication. Biological Psychiatry, 61(3), 348-358.

Jeffery, R.W., Drewnowski, A., Epstein, L.H., Stunkard, A.J, Wilson, G.T., Wing, R.R, \& Hill, D.R. (2000). Long-term maintenance of weight loss: current status. Health Psychology, 19(1), 5-16.

Kopelman, P. (2007). Health risks associated with overweight and obesity. Obesity Reviews, 8, 13-17.

Kaly, P., Orellana, S., Torrella, T., Takagishi, C., Saff-Koche, L., \& Murr, M. M. (2008). Unrealistic weight loss expectations in candidates for bariatric surgery. Surgery for Obesity and Related 
Diseases: The Official Journal of the American Society for Metabolic and Bariatric Surgery, $4(1), 6-10$.

Krijn, M., Emmelkamp, P. M. G., Olafson, R. P., \& Biemond, R. (2004). Virtual reality exposure therapy of anxiety disorders: A review. Clinical Psychology Review, 24, 259-281.

Lenggenhager, B., Tadi, T., Metzinger, T., \& Blanke, O. (2007). Video ergo sum: manipulating bodily self-consciousness. Science, 317(5841), 1096-1099.

Lumeng, J.C., Gannon, K., Cabral, H.J., Frank, D.A., \& Zuckerman B.(2003). Association between clinically meaningful behavior problems and overweight in children. Pediatrics, 112(5), 11381145.

Maher, N. E., \& Gold, J. I. (2009). The use of virtual reality for pain control: a review. Current Pain and Headache Reports, 13(2), 100-109.

Mann, T., Tomiyama, A.J., Westling, E., Lew, A.M., Samuels, B., \& Chatman, J. (2007). Medicare's search for effective obesity treatments: diets are not the answer. American Psychology, 62(3), $220-233$.

Manzoni, G.M., Gorini, A., Preziosa, A., Pagnini, F., Castelnuovo, G., Molinari, E., \& Riva, G. (2008). New technologies and relaxation: An explorative study on obese patients with emotional eating. Journal of Cybertherapy and Rehabilitation, 1, 182-192.

Manzoni, G.M., Pagnini, F., Gorini, A., Preziosa, A., Castelnuovo, G., Molinari, E., \& Riva, G. (2009). Can Relaxation Training Reduce Emotional Eating in Women with Obesity? An Exploratory Study with 3 Months of Follow-Up. Journal of the American Dietetic Association, 109, 14271432.

Mokdad, A.H., Ford, E.S., Bowman, B.A., Dietz, W.H., Vinicor, F., Bales, V.S., \& Marks, J.S. (2001). Prevalence of obesity, diabetes, and obesity-related health risk factors. Journal of the American Medicine Association, 289(1), 76-79. 
Neumark-Sztainer, D. (2003). Obesity and Eating Disorder Prevention: An Integrated Approach. Adolescent Medicine, 14(1), 159-173.

Parsons, T. D., \& Rizzo, A. A. (2008). Affective outcomes of virtual reality exposure therapy for anxiety and specific phobias: a meta-analysis. Journal of Behavior Therapy and Experimental Psychiatry, 39, 250-261.

Perpiñá, C., Baños, R., Botella, C., \& Marco, J. H. (2001). Virtual reality as a therapy tool: A case study on body image alteration disorders. Revista Argentina de Clínica Psicológica, 10, 227-241.

Perpiñá, C., Botella C., \& Baños R. M. (2003). Virtual reality in eating disorders. European Eating Disorders Review, 11, 261-278.

Perpiñá, C., Botella, C., \& Baños, R. M. (2000). Imagen Corporal en los Trastornos Alimentarios. Evaluación y Tratamiento Mediante Realidad Virtual. Valencia: Promolibro.

Perpiñá, C., Botella, C., Baños, R. M., Marco, H., Alcañiz, M., \& Quero, S. (1999). Body image and virtual reality in eating disorders: is exposure to virtual reality more effective than the classical body image treatment? Cyberpsychology and Behavior, 2, 149-159.

Perpiñá, C., Marco, J. H., Botella, C., \& Baños, R. (2004). Tratamiento de la imagen corporal en los trastornos alimentarios mediante tratamiento cognitivo-comportamental apoyado con realidad virtual: resultados al año de seguimiento. Psicología Conductual: Revista Internacional de Psicología Clínica y de la Salud, 12, 519-537.

Poirier, P., Giles, T.D., Bray, G.A., Hong, Y., Stern, J.S., Pi-Sunyer, F.X., \& Eckel, R.H. (2006). Obesity and cardiovascular disease: pathophysiology, evaluation, and effect on weight loss. Arteriosclerosis, Thrombosis, and Vascular Biology, 26(5), 968-976.

Powers, M. B., \& Emmelkamp, P. M. G. (2008). Virtual reality exposure therapy for anxiety disorders: A meta-analysis. Journal of Anxiety Disorders, 22, 561-569.

Riva, G. (1998). Virtual environments for body image modification: Virtual reality system for the treatment of body image disturbances. Computers in Human Behavior, 14, 477-490.

Riva, G. (2005). Virtual reality in psychotherapy: Review. CyberPsychology \& Behavior, 8(3), 220-230. 
Riva, G. (2007, 8-10 October). Virtual Body, Real Pain: The Allocentric Lock Hypothesis. Paper presented at the Body Representation Workshop, Rovereto, Italy.

Riva, G. (2011). The Key to Unlocking the Virtual Body: Virtual Reality in the Treatment of Obesity and Eating Disorders. Journal of Diabetes Science and Technology, 5(2), 283-292.

Riva, G. (2012). Neuroscience and eating disorders: The allocentric lock hypothesis. Medical Hypotheses, $78,254-257$.

Riva, G., Bacchetta, M., Baruffi, M., \& Molinari, E. (2001). Virtual reality-based multidimensional therapy for the treatment of body image disturbances in obesity: A controlled study. Cyberpsychology \& Behavior, 4, 511-526.

Riva, G., Bacchetta, M., Baruffi, M., \& Molinari, E. (2002). Virtual-reality-based multidimensional therapy for the treatment of body image disturbances in binge eating disorders: a preliminary controlled study. IEEE transactions on information technology in biomedicine: a publication of the IEEE Engineering in Medicine and Biology Society, 6(3), 224-234.

Riva, G., Bacchetta, M., Baruffi, M., Cirillo, G., \& Molinari, E. (2000). Virtual reality environment for body image modification: A multidimensional therapy for the treatment of body image in obesity and related pathologies. CyberPsychology \& Behavior, 3, 421-431.

Riva, G., Bacchetta, M., Baruffi, M., Cirillo, G., \& Molinari, E. (2000). Virtual Reality Environment for Body Image Modification: A Multidimensional Therapy for the Treatment of Body Image in Obesity and Related Pathologies. CyberPsychology \& Behavior, 3(3), 421-431

Riva, G., Bacchetta, M., Baruffi, M., Rinaldi, S., \& Molinari, E. (1998). Experiential cognitive therapy in anorexia nervosa. Eating and weight disorders, 3, 141-150.

Riva, G., Bacchetta, M., Baruffi, M., Rinaldi, S., \& Molinari, E. (1999). Virtual reality based experiential cognitive treatment of anorexia nervosa. Journal of Behavior Therapy and Experimental Psychiatry, 30, 221-230.

Riva, G., Bacchetta, M., Baruffi, M., Rinaldi, S., Vincelli, F., \& Molinari, E. (2000). Virtual reality based experiential cognitive treatment of obesity and binge-eating disorders. Clinical Psychology and Psychotherapy, 7, 209-219. 
Riva, G., Bacchetta, M., Cesa, G., Conti, S., \& Molinari, E. (2003). Six-month follow-up of in-patient experiential-cognitive therapy for binge eating disorders. CyberPsychology \& Behavior, 6, $251-258$

Riva, G., Bacchetta, M., Cesa, G., Conti, S., Castelnuovo, G., Mantovani, F., \& Molinari, E. (2006). Is severe obesity a form of addiction? Rationale, clinical approach, and controlled clinical trial. CyberPsychology and Behavior, 9, 457-479.

Riva, G., Carelli, L., Gaggioli, A., Gorini, A., Vigna, C., Corsi, R.,. . . Vezzadini, L. (2009). NeuroVR 1.5 - a free virtual reality platform for the assessment and treatment in clinical psychology and neuroscience. Studies in health technology and informatics, 142, 268-270.

Riva, G., Castelnuovo, G., Cesa, G., Gaggioli, A., Mantovani, F., \& Molinari, E. (2012). Virtual Reality for Enhancing the Cognitive Behavioral Treatment of Obesity: A Controlled Study with OneYear Follow-up. Paper presented at the Medicine 2.0'12 Boston. Online: http://www.medicine20congress.com/ocs/index.php/med/med2012/paper/view/1197

Riva, G., Gaggioli, A., Gorini A., Carelli, L., Repetto C., Algeri D., \& Vigna C. (2009). Virtual Reality as Empowering Environment for Personal Change. Anuario de Psicologia, 40(2), 171-192.

Riva, G., Gaggioli, A., Grassi, A., Raspelli, S., Cipresso, P., Pallavicini, F., . . Donvito, G. (2011). NeuroVR 2 - A Free Virtual Reality Platform for the Assessment and Treatment in Behavioral Health Care. Studies in health technology and informatics, 163, 493-495.

Riva, G., Gaggioli, A., Villani, D., Preziosa, A., Morganti, F., Corsi, R., . . Vezzadini, L. (2007). NeuroVR: an open source virtual reality platform for clinical psychology and behavioral neurosciences. Studies in health technology and informatics, 125, 394-399.

Riva, G., \& Gaudio, S. (2012). Allocentric lock in anorexia nervosa: New evidences from neuroimaging studies. Medical Hypotheses, 79(1), 113-117.

Riva, G., \& Melis (1997a).Virtual reality for the treatment of body image disturbances. In G. Riva (Ed.), Virtual Reality in Neuro-Psycho-Physiology: Cognitive, clinical and methodological issues in the assessment and rehabilitation (pp. 95-111). Amsterdam: IOS Press. 
Riva, G., \& Melis (1997b).Virtual reality for the treatment of body image disturbances. Studies in Health Technology and Informatics, 44, 95-111.

Riva, G., Melis, L., \& Bolzoni, M. (1997). Treating Body image disturbances. Communications of the $A C M, 40,69-71$.

Salorio del Moral, P., Gómez Sánchez, R., Morales Moreno, I., Torres Ortuño, A., Díaz Cuenca, A., \& Alegría Capel, A. (2004). La realidad virtual, una nueva herramienta terapéutica. Tratamiento de la imagen corporal en los trastornos alimentarios. Revista Electrónica Semestral de Enfermería, 5, 1-17. Retrieved from http://revistas.um.es/eglobal/article/view/543/563.

Slater, M., Spanlang, B., Sanchez-Vives, M. V., \& Blanke, O. (2010). First person experience of body transfer in virtual reality. PLoS One, 5(5), e10564. doi: 10.1371/journal.pone.0010564

Sorbara, M., \& Geliebter, A. (2002). Body image disturbance in obese outpatients before and after weight loss in relation to race, gender, binge eating, and age of onset of obesity. The International journal of eating disorders, 31(4), 416-423.

Staiano, A. E., \& Calvert, S. L. (2011). Exergames for Physical Education Courses: Physical, Social, and Cognitive Benefits. Child Development Perspectives, 5, 93-98.

Spagnolli., A., Gamberini., L. (2005). A Place for Presence. Understanding the Human Involvement in Mediated Interactive Environments. PsychNology Journal, 3(1), 6-15. On-line: http://www.psychnology.org/index.php?page=abstract---volume-3---spagnolli

Thorburn, A.W. (2005). Prevalence of obesity in Australia. Obesity Reviews, 6, 187-189.

Vander Wal, J.S., \& Thelen, M.H. (2000). Eating and Body Image Concerns Among Obese and AverageWeight Children. Addictive Behavior, 25(5), 775-778.

WHO (2012). Obesity and overweight. World Health Organization Fact Sheet No. 311. http://www.who.int/mediacentre/factsheets/fs311/en/index.html, accessed January 3, 2013.

Wilson, G. T., Grilo, C. M., \& Vitousek, K. M. (2007). Psychological treatment of eating disorders. American Psychologist, 62, 199-216. 
Yanovski, S.Z. (2002). Binge Eating in Obese Persons. In Fairburn, C.G., Brownell, K.D. (eds), Eating Disorders and Obesity, 2nd ed. New York: Guilford Press, 403-407.

Zimmerman, T. G., Lanier, J., Blanchard, C., Bryson, S., \& Harvill, Y. (1986). A Hand Gesture Interface Device. ACM SIGCHI Bulletin, 189-192.

Accepted Version 
Table 1. Criteria for searches on the use of VR in the treatment of ED, BED, and obesity.

\begin{tabular}{lc}
\hline Category Criteria &
\end{tabular}

\begin{tabular}{ll}
\hline Study population & Patients with a diagnosis of ED (AN, BN, EDNOS, and BED) or \\
obesity
\end{tabular}

Study setting

Time period

Study design and other criteria
All nations

From 1986 to 2012

Included:

- Original research studies (non-controlled and randomized controlled trials)

- Case studies

Excluded:

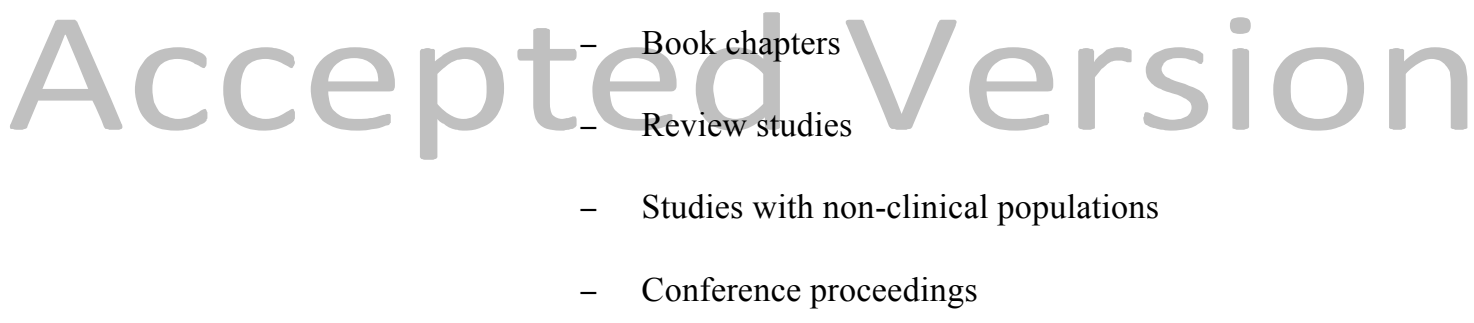


Table 2. Studies about the effectiveness of VR-based treatments for ED and obesity

\begin{tabular}{|c|c|c|c|c|c|}
\hline Author(s), year & Condition(s) & $\begin{array}{l}N \text { (total); } N \\
\text { dropouts }\end{array}$ & Sessions & Dependent variables & Outcome (effectiveness) \\
\hline \multicolumn{6}{|l|}{ Case studies } \\
\hline $\begin{array}{l}\text { Riva, Bacchetta, Baruffi, Rinaldi \& } \\
\quad \text { Molinari }(1998,1999)\end{array}$ & 1 VEBIM 2 & $\begin{array}{l}\text { 22-year-old female } \\
\text { with AN binge- } \\
\text { eating/purging subtype }\end{array}$ & 8 VEBIM-2 sessions. & BIAQ, BSS, CDRS, FRS & $\begin{array}{l}\text { Increased bodily awareness. } \\
\text { Reduction of BI dissatisfaction. } \\
\text { High degree of motivation to } \\
\text { change. }\end{array}$ \\
\hline $\begin{array}{l}\text { Perpiñá, Baños, Botella, \& Marco } \\
\text { (2001) }\end{array}$ & $1 \mathrm{CBT}+\mathrm{VR}$ & $\begin{array}{l}\text { 22-year-old female } \\
\text { with AN }\end{array}$ & $\begin{array}{l}\text { CBT: } 8 \text { group sessions }+ \\
\text { VR: } 6 \text { individual parallel } \\
\text { sessions to CBT }\end{array}$ & $\begin{array}{l}\text { BSQ, BIATQ, SIBID, Body } \\
\text { Acceptance Scale, EAT-40, } \\
\text { EDI-II, BSI }\end{array}$ & $\begin{array}{l}\text { Reduction of body dissatisfaction, } \\
\text { reduction of negative thoughts } \\
\text { about body image, reduction of } \\
\text { negative attitudes towards the body, } \\
\text { reduction of drive for thinness and } \\
\text { interoceptive awareness, and } \\
\text { reduction of situations related to } \\
\text { body image discomfort. } \\
\text { Six-month and one-year follow-up } \\
\text { data: improvement of treatment } \\
\text { achievements. }\end{array}$ \\
\hline $\begin{array}{l}\text { Salorio del Moral, Gómez Sánchez, } \\
\text { Morales Moreno, Torres Ortuño, } \\
\text { Diaz Cuenca, \& Alegría Capel } \\
\text { (2004) }\end{array}$ & $\begin{array}{l}1 \mathrm{CBT}+\text { "Virtual \& } \\
\text { Body" VR software } \\
\text { (Perpiñá, Botella, \& } \\
\text { Baños, 2000) }\end{array}$ & $\begin{array}{l}1 \text { teenage girl with AN } \\
\text { restricting subtype }\end{array}$ & $\begin{array}{l}\text { CBT: } 15 \text { group sessions } \\
+ \\
\text { VR: } 10 \text { individual parallel } \\
\text { sessions to CBT }\end{array}$ & $\begin{array}{l}\text { EDI-2, BES, BSQ, BIAQ, } \\
\text { weight, Test in the Mirror, BI } \\
\text { weekly control, and ideal, real, } \\
\text { and subjective body image } \\
\text { discrepancies during VRE. }\end{array}$ & $\begin{array}{l}\text { Improvement of body satisfaction, } \\
\text { increased interoceptive awareness, } \\
\text { decreased perfectionism, and no } \\
\text { asceticism tendency. } \\
\text { One-year follow-up data: No drive } \\
\text { for thinness, greater body }\end{array}$ \\
\hline
\end{tabular}


satisfaction, higher interpersonal trust, no perfectionism, asceticism tendency or social insecurity, and decrease in general

symptomatology.

1-year follow-up: No disturbances in either body image or weight.

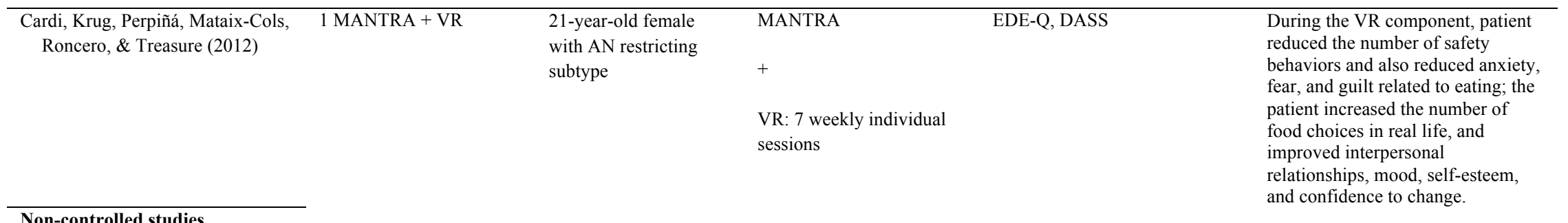

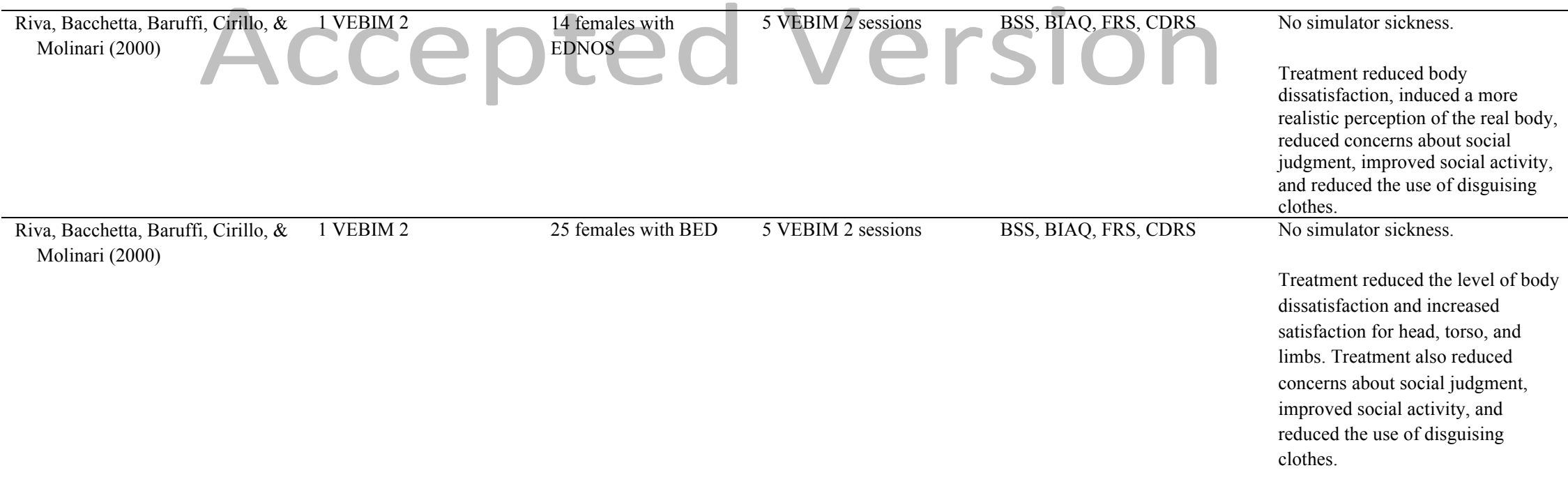

Riva, Bacchetta, Baruffi, Rinaldi,

25 females with BED

Session 1: Exposure to

, EDI-2, BSS, BIAQ,

Body dissatisfaction level was

VEBIM 2 (assessment of FRS, CDRS 
Sessions 2-4: Exposure to

VEBIM $2+$ Butters and

Cash therapy (1987)

(assessment and

modification of symptoms

of anxiety related to food

exposure, and the body

experience of the

participant)
Improvement in social activity and reduced use of disguising clothes.

No simulator sickness.

\begin{tabular}{ll}
\hline Controlled studies & \\
\hline $\begin{array}{l}\text { Perpiñá, Botella, Baños, Marco, } \\
\text { Alcañiz, \& Quero (1999) }\end{array}$ & 2 (between subject): \\
& $\begin{array}{l}\text { SBIT (BI treatment } \\
\text { adapted from Cash [1996] } \\
\text { and Rosen [1997]+ } \\
\text { relaxation) }\end{array}$ \\
$\&$ \\
VR (BI treatment adapted \\
from Cash [1996] and \\
Rosen [1997] + VR)
\end{tabular}

18 ED females.

At the end there

remained 5 in the SBIT

condition ( $3 \mathrm{AN} \& 2$

$\mathrm{BN})$ and 8 in the $\mathrm{VR}$

condition (4 AN \& 4

$\mathrm{BN})$; there were 5

dropouts

Rosen
SBIT: 3 weekly group

sessions of 3 hours +6

BDI, PANAS, EAT, RS, BITE,

1 hour p

1 hour.

VR: 3 weekly group

sessions of 3 hours +6

weekly parallel sessions of

1 hour.

\section{Body interference, fear of}

putting on weight, self-rating

scales to assess VR sessions. the two conditions on general ED measures

Participants in the VR condition

experienced a high degree of realism in the VE. 


$\begin{array}{llll}\begin{array}{l}\text { Riva, Bacchetta, Baruffi, \& } \\ \text { Molinari (2002) }\end{array} & \begin{array}{l}\text { 2 (between subject): } \\ \text { VREDIM (+ low-calorie } \\ \text { diet and physical training) }\end{array} & \begin{array}{l}\text { 20 BED women (10 } \\ \text { VREDIM, 10 PNI) }\end{array} & \begin{array}{l}\text { Experimental group: 7 } \\ \text { VREDIM sessions (+/-6.5 } \\ \text { weeks). }\end{array} \\ & \begin{array}{l}\text { Control group: 3 PNI } \\ \text { PNI (+ low-calorie diet } \\ \text { and physical training) }\end{array} & \begin{array}{l}\text { sessions a week (+/-6.5 } \\ \text { weeks). }\end{array}\end{array}$

DIET, STAI, AI, WELSQ,

URICA, BSS, BIAQ, FRS,

CDRS

weeks).
VREDIM group showed 1) a

significant reduction in body

dissatisfaction, overeating, and

anxiety; 2) a significant increase in

self-efficacy and motivation to

change; and 3) improvement in

social activities.

PNI group only showed a

significant reduction in anxiety

after treatment (only on AI but not on STAI).

No differences in the reduction of BE behavior.

(No follow-up results)

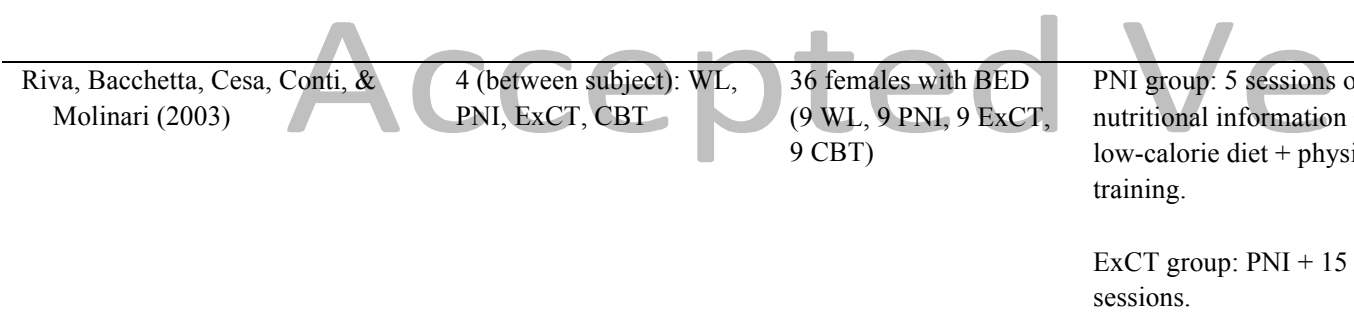

CBT: PNI +15 sessions.

\section{DIET, STAI, BDI-II, RAS \\ RSEQ \\ BIAQ, CDRS \\ State anxiety significantly
decreased in both the ExCT and \\ PNI groups.}

Depression significantly decreased

in both the ExCT (complete

remission) and $\mathrm{CBT}$ groups.

Self-esteem improved in all groups, except for $\mathrm{WL}$.

Positive changes in terms of

assertive behaviors in ExCT only.

Eating control and eating self-

efficacy significantly increased in

all conditions except for WL.

ExCT was more effective than CBT in improving BI (body awareness, 
body satisfaction, and physical acceptance).

Weight loss was similar in all conditions except for WL.

Six-month follow-up: $77 \%$ quit binging for ExCT vs. $56 \%$ for CBT and $22 \%$ for PNI.

Perpiñá, Marco, Botella, \& Baños 2 (between subject): (2004)
SBIT \&

$\mathrm{CBT}+\mathrm{VR}$
$5 \mathrm{BN})$
3 weekly group treatment (Cash and Rosen) sessions of 3 hours +6 weekly parallel VR sessions of 1

hour.
BDI, PANAS, EAT, RS, BITE, EDI-2, BSQ, BIAQ, BAT, BIATQ, ASI, SIBID, BASS, Body interference, fear of putting on weight, self-rating scales to assess VR sessions. The combination of CBT and VR increased the power of the results of

SBIT. Results at post-treatment improved over 1 year and extended to ED and general psychopathology.

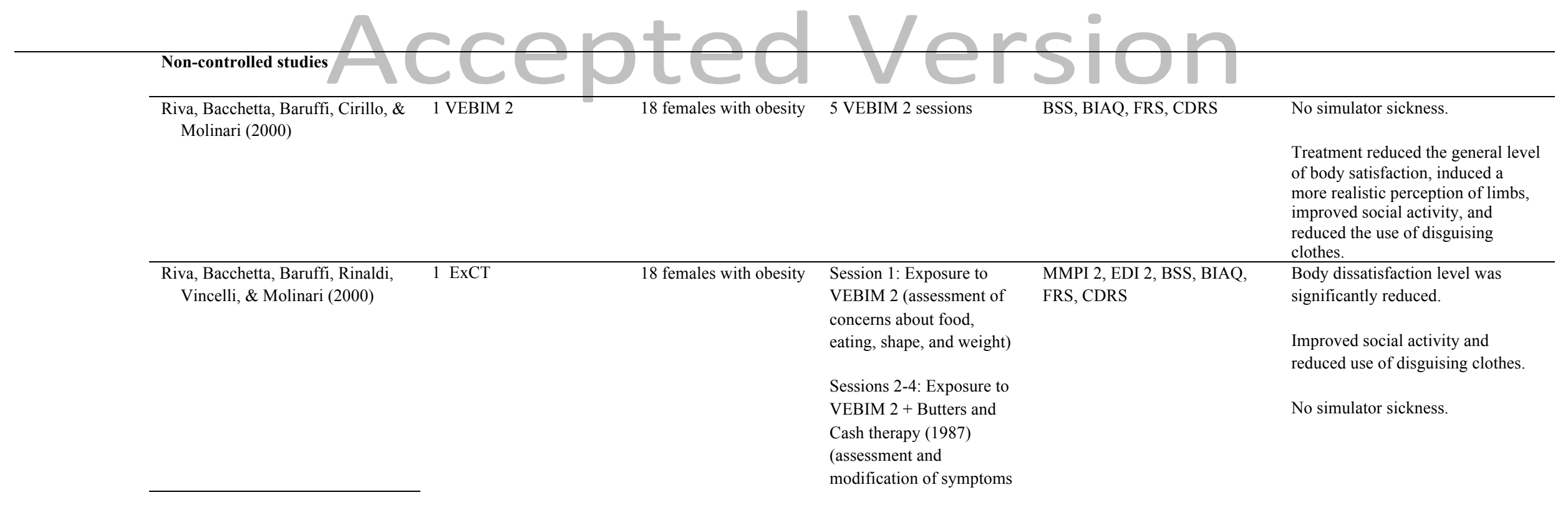


of anxiety related to food

exposure, and the body

experience of the

participant)

\section{Controlled studies}

\begin{tabular}{|c|c|c|c|c|}
\hline \multirow[t]{2}{*}{$\begin{array}{l}\text { Riva, Bacchetta, Baruffi, \& } \\
\text { Molinari (2001) }\end{array}$} & $\begin{array}{l}2 \text { (between subject): } \\
\text { VREDIM (+ low-calorie } \\
\text { diet and physical training) } \\
\&\end{array}$ & $\begin{array}{l}28 \text { women with obesity } \\
\text { (14 VREDIM, } 14 \text { PNI) }\end{array}$ & $\begin{array}{l}\text { Experimental group: } 7 \\
\text { VREDIM sessions (+/-6.5 } \\
\text { weeks). }\end{array}$ & $\begin{array}{l}\text { DIET, STAI, AI, WELSQ, } \\
\text { URICA, BSS, BIAQ, FRS, } \\
\text { CDRS }\end{array}$ \\
\hline & PNI (+ low-calorie c & & $\begin{array}{l}\text { Control group: } 3 \text { PNI } \\
\text { sessions per week (+/-6.5 } \\
\text { weeks). }\end{array}$ & \\
\hline
\end{tabular}

No simulator sickness in VREDIM group.

VREDIM was more effective than PNI in improving body satisfaction,

self-efficacy, and motivation to

change (although difference in thi

last aspect was not significant).

VREDIM group significantly reduced body dissatisfaction, anxiety and overeating, and significantly increased self-efficacy after treatment. PNI group only

significantly reduced anxiety (as measured with the AI but not with the STAI) and increased exercising. Mean weight reduction of $11.33 \mathrm{~kg}$

in VREDIM group vs. $7.58 \mathrm{~kg}$ in

PNI group (no significant

differences).

(No follow-up results) 
CBT: PNI + 15 sessions

over 6 weeks.

ExCT: PNI +15 sessions over 6 weeks.
In general, the reduction in anxiety, eating behavior characteristics, and body dissatisfaction was greater, though non-significantly, in the ExCT condition.

At 6-month follow-up, ExCT was the only approach able to

significantly improve both the level of BI satisfaction and self-efficacy, which implies a reduction in which inplios a reduction in

improvement in adaptive behaviors.

\begin{tabular}{lll}
\hline $\begin{array}{l}\text { Manzoni, Gorini, Preziosa, } \\
\text { Pagnini, Castelnuovo, Molinari, } \\
\text { \& Riva (2008) }\end{array}$ & $\begin{array}{l}\text { 3 (between subject): VR } \\
\text { (relaxation protocol } \\
\text { enhanced by VR); IM } \\
\text { (relaxation protocol using } \\
\text { imagery); WL (waiting }\end{array}$ & $\begin{array}{l}\text { 60 females with obesity } \\
\text { (20 VR, 20 IM, 20 }\end{array}$ \\
\hline list)
\end{tabular}

VR and IM: 5-week

hospital-based program for

BDI, WELSQ, STAI, VAS,

ight reduction and

HR, weight

rehabilitation (5 sessions)

+ 3-week relaxation

program (12 sessions).

WL: 5-week hospital-

based program for weigh

reduction and

rehabilitation

perceived self-efficacy for eating state anxiety, increased the level of
VR and IM conditions increased

control and reduced the level of

depression. These changes were not found in the WL condition.

VR and IM conditions also reduced relaxation, and reduced heart rate during exposure to stressful situations.

No differences between VR and IM were found.

Weight significantly decreased in all the conditions.

\begin{tabular}{cl}
\hline Manzoni, Pagnini, Gorini, & 3 (between subject): VR \\
Preziosa, Castelnuovo, Molinari, & (relaxation protocol \\
\& Riva (2009) & enhanced by VR); IM \\
& (relaxation protocol using \\
imagery); CTRL (control \\
condition)
\end{tabular}

$\begin{array}{lll}60 \text { females with obesity } & \text { VR and IM: 5-week } & \text { BDI, WELSQ, STAI, EOQ, } \\ \text { (20 VR, 20 IM, 20 } & \text { hospital-based program for } & \begin{array}{l}\text { weight } \\ \text { CTRL) }\end{array} \\ & \text { weight reduction and } & \\ & \text { rehabilitation (5 sessions) } \\ & +3 \text {-week relaxation } & \\ & \text { program (12 sessions). }\end{array}$

CTRL: 5-week hospital-
Emotional eating episodes fell to

zero and weight significantly

decreased in all groups.

VR and IM condition improved self-efficacy for eating control and depression, whereas no significant change was found in the CTRL 
Treatment: CBT (cognitive-behavioral therapy), ExCT (experiential cognitive therapy: based on VR), MANTRA (Maudsley Model of Treatment for Adults with Anorexia Nervosa), PNI (psychonutritional intervention based on cognitive-behavioral approach), SBIT (standard BI treatment), VEBIM (Virtual Environments for Body Image Modification), VEBIM 2 (enhanced version of VEBIM), VR (virtual reality), VRE (virtual reality exposure), VREDIM (Virtual Reality for Eating Disorders Modification: Improved version of VEBIM), WL (waiting list)

Dependent variables: AI (Assertion Inventory), ASI (Appearance Schemas Inventory), BASS (Body Areas Satisfaction Scale), BAT (Body Attitudes Test), BDI (Beck Depression Inventory), BDI-II (Beck Depression Inventory-II), BES (Body Esteem Scale), BIAQ (Body Image Avoidance Questionnaire), BIATQ (Body Image Automatic Thoughts), BITE (Bulimic Investigatory Test Edinburgh); BSQ (Body Shape Questionnaire), BSS (Body Satisfaction Scale), CDRS (Contour Drawing Ratings Scale), DASS (Depression, Anxiety, and Stress Scale), DIET (Dieter's Inventory of Eating Temptations), EAT (Eating Attitudes Test), EDE-Q (Eating Disorders Examination

Questionnaire), EDI-2 (Eating Disorders Inventory-2), EOQ (Emotional Overeating Questionnaire), FRS (Figure Rating Scale), HR (heart rate); MMPI-2 (Minnesota Multiphasic Personality Inventory 2), PANAS (Positive and Negative Affect Schedule), RAS (Rathus Assertiveness Schedule), RS (Restrained Scales), RSEQ (Rosenberg Self-Esteem Questionnaire), SIBID (Situational Inventory of Body Image Dysphoria), STAI (State-Trait Anxiety Inventory), URICA (University of Rhode Island Change Assessment Scale), VAS (visual analogue scale), WELSQ (Weight Efficacy Life-Style Questionnaire) 